\title{
Medicolegal Importance of the Role of Ubiquitin Expression in Suprarenal and Renal Tissues in Blunt and Sharp Traumatic Deaths: Immunohistochemical Study
}

\author{
Ayman Nagy, Ghada El-Sarngawy and Karima El-Desoky ${ }^{1}$
}

${ }^{1}$ Forensic and Clinical Toxicology Department \& Pathology Department, Faculty of Medicine- Tanta University.

\begin{abstract}
Introduction: Injuries and violence represent a major public health problem worldwide. It is still a leading cause of mortality specially in developing countries. Ubiquitin is a heat shock protein, which is induced by various kinds of traumatic stress. Aim of the work: The aim of this study is to investigate the immunohistochemical expression of ubiquitin in suprarenal and renal tissues in blunt and sharp traumatic deaths. Subjects and methods: Cross sectional post-mortem study was conducted on the traumatic medicoegal autopsy cases and natural cases in Banha medicolegal unit (Ministry of Justice) over six months interval from February 2016 to August 2016. For all medicolegal cases, the data collection sheet included age, sex, date of death, survival time and cause of death. Histological examinations and ubiquitin immunostaining were performed on the suprarenal and renal tissues. Results: Forty-five autopsy cases were included in this study. The majority of victims were males $(84.4 \%)$ from rural regions $(70.5 \%)$. Blunt injuries represented the majority of traumatic deaths $(57.14 \%)$. The greater part of traumatic cases $(74.3 \%)$ died within 24 hours post injuries. Traumatic cases revealed acute tubular necrosis and vascular degeneration in renal tissue and exhaustion of lipid contents of zona fasiculata in suprarenal tissue specimens. Traumatic deaths showed significant increase in ubiquitin expression in compared to natural deaths in renal distal convoluted tubules and suprarenal cortex. Moreover, there was significant positive correlation between survival period and positive ubiquitin immune expression in each of renal distal convoluted tubules $(\mathrm{r}=0.461, \mathrm{p}=0.005)$ and suprarenal cortex $(\mathrm{r}=0.459, \mathrm{p}=0.006)$. Conclusion: Ubiquitin immunoexpression can serve as a stress indicator of traumatic deaths and it may be useful for determination of survival period.
\end{abstract}

Keyword Ubiquitin, traumatic deaths, Traumatic stress, kidney, suprarenal, immunohistochemistry, autopsy cases

\section{Introduction}

Worldwide, trauma is considered one of the major causes of death and disability especially in developing countries (Mahran et al., 2016).It represents the third cause of death for all ages (account for $9 \%$ of annual mortality) (Krug et al. 2000). According to WHO reports, trauma is expected to be the leading cause of death by 2020 (Pfeifer et al., 2009; Kleber et al., 2013). In Egypt, traumatic deaths represented the fifth leading cause of death in 2004 and accounted for 8\% from all deaths (Mahran et al., 2013).

Severe trauma is associated with organs dysfunction with integrated metabolic response (Patel et al., 2007). Many previous reports demonstrated renal and adrenal gland injuries after severe trauma (De Abreu et al., 2010; Roupakias et al., 2012 and lin and $\mathrm{Wu}, 2013)$.As severe trauma or crush injuries are usually associated with severe stress and decreased renal perfusion and rhabdomyolysis (Stene, 1990; Morris et al., 1991).

Histologically, the suprarenal gland consists of cortex and medulla. The cortex divided into three zones. The outer zone is zona glomeruloza which is the site of mineralocorticoids secretion. The inner zone called zona reticularies which lies directly next to the medulla and involved in the secretion of glucocorticoids and sex hormones (especially androgen). Zona Fasiculata represents about $80 \%$ of cortex tissues and lies between the above mentioned two zones. It secrets glucocorticoids and adrenal androgen. While, the adrenal medulla lies in the innermost part of adrenal gland and surrounded by the cortex and it is responsible for epinephrine and norepinephrine secretion in response to sympathetic stimulation (Ross and Pawlina, 2011).

Ubiquitin is a specific protease; with 8.500 molecular weight. This protein presents in all euokaryocytes, which is expressed intracellular and extracellar areas. It has important intracellular functions as heat shock response, protein breakdown and immune response regulation. Additionally, it has an antiinflammatory function by its cytokine like protein properties (Guler et al., 2011).

Ubiquitin is a common immunohistochemical marker, which, has been studied in different types of traumatic stress in forensic pathology as in fire 
fatalities, brain injuries and traumatic deaths (Hausmann et al., 1999; Quan et al., 2001b; Quan et al., 2005 and Ishikawa et al., 2007).

There are a little data of ubiquitin marker for investigating systemic stress response in traumatic deaths. Therefore, the aim of this study is to investigate the immunohistochemical expression of ubiquitin in suprarenal and renal tissues in blunt and sharp traumatic deaths.

\section{Subjects and Methods}

\section{Study design and ethical consideration:}

It is a cross sectional post-mortem study on traumatic medicolegal autopsy cases and natural cases. It was carried out at Faculty of Medicine Tanta University during the period from February 2016 to August 2016. All cases were retrieved from Banha medicolegal unit (Ministry of Justice). The study was approved by Research Ethics committee of Tanta Faculty of Medicine. The consent for taken samples of all cases was approved by Banha medicolegal unit. Appropriate precautions during autopsy were applied and confidentiality of records was conserved by keeping the records anonymous.

\section{Subjects:}

All traumatic causes of death (sharp injuries and blunt injuries) and natural deaths (deaths from natural causes other than trauma and poisons) with postmortem interval less than 48 hours were included in this study. Autopsy of natural deaths was done in suspicious cases, if it suddenly occurred in apparent healthy subjects to exclude the likelihood of unnatural death (Chaudhari et al., 2013). Decomposed bodies, cases with renal, suprarenal trauma and cases with diseases that could alter ubiquitin as chronic observed liver \& chronic renal pathological changes at autopsy were excluded (Sixt and Dahlmann, 2008; Wang and Mitch, 2014). For all medicolegal cases, the data collection sheet included age, sex, date of death, survival time and causes of death (sharp injuries, blunt injuries or natural deaths) was performed.

\section{Methods:}

\section{Tissue sections:}

Post mortem dissection was done and carefully kidney and suprarenal gland were excised immediately and fixed in neutral buffered $10 \%$ formaldehyde and routinely processed. Paraffin impeded sections $(5 \mu \mathrm{m}$ thickness) were done for hematoxylin and eosin (H\&E) staining and immunohistochemical staining for ubiquitin. Immunohistochemistry staining for ubiquitin:

Immunohistochemical staining for Ubiquitin (Ub) was performed on formalin fixed paraffin embedded suprarenal and kidney sections. Polyclonal rabbit anti-human ubiquitin antibody (My Bio source, USA; Catalog Number; MBS280030) was used at a 100 -fold dilution with $3 \mathrm{~h}$ incubation at $37{ }^{\circ} \mathrm{C}$, according to the manufacturer's instructions (counterstaining with hematoxylin).

Quantitative analysis of ubiquitin in renal and suprarenal tissues:

Expression of Ubiquitin__staining was considered positive, if there was nuclear and cytoplasmic or memberanous brownish staining. The total numbers of positively immunereactivity cells were counted under 200 magnification: five random fields were examined for each structure (proximal, distal tubules\& collecting ducts for renal tissue and adrenal cortex and medulla). The percentages of ubiquitin positivity were estimated by number of positive epithelial cells/total number of epithelial cells x 100 . Then the percentages of ubiquitin positivity were scored as: grade 0 indicated no expression; + Mild positivity (Grade I) if it was $<20 \%$ brownish staining, ++ Moderate positivity (Grade II) if it was $<40 \%$ positivity and +++ Extensive positive (Grade III) if more than $50 \%$ positivity.

Statistical analysis:

Statistical analysis and presentation of data was conducted using SPSS (Statistical Package for the Social Science) version 20 computer program. Qualitative data were expressed as number and percentages in brackets and Pearson's Chi square test was used to examine association between two variables. For quantitative data, the Shapiro-Wilk test for normality was performed and ANOVA and Kruskal-Wallis tests were used for comparison between the studied and control groups. In addition, Pearson's correlation between ubiquitin expression and survival period was analyzed. Significance was adopted at $\mathrm{p}<0.05$ for interpretation of results of tests (Dawson-Saunders and Trapp, 2001).

\section{Results}

Demographics and deaths circumstances:

Forty-five autopsy cases were included in this study. Their age ranged from 20 to 70 years with a mean of $43.89 \pm 13.02$ years. The majority of victims were males $(84.4 \%)$ from rural regions $(70.5 \%)$. Traumatic deaths accounted for 35 cases $(77.77 \%)$ while natural deaths accounted for 10 cases $(33.33 \%)$ of all autopsy cases. Of all traumatic cases, blunt injuries represented 20 cases $(57.14 \%)$ and the remaining 15 cases $(42.86 \%)$ belonged to sharp injuries. Regarding survival time, the majority of traumatic cases $(74.3 \%$ ) died within 24 hours post injuries; where $80 \%$ of cases with fatal sharp injuries and $70 \%$ of blunt injuries victims died within 24 hours. There were no significant difference between natural deaths and traumatic deaths as regards age, sex and residence. Additionally, no significant difference was detected between blunt and sharp cases regarding survival period (Table 1).

Histopathological results of hematoxylin and eosin (H\&E) staining:

Histological examination of sections from suprarenal gland of natural death demonstrated normal histopathological picture of the three zones of the gland (zona glomeruloza, zona fasiculata and zona reticularies) with mild edema in between parenchymal cells (Fig.1). Meanwhile, all cases of traumatic deaths showed exhaustion of lipid contents of zona fasiculata with decrease its cell size (Fig.2).

Examination of H\&E stained sections of renal specimens revealed only cloudy swelling of proximal convoluted tubules of the kidney together with slight widening of bowman space in all cases of natural deaths (Fig.3). However, the traumatic cases revealed acute tubular necrosis in proximal convoluted tubules and vascular degeneration in distal tubules (Fig.4). 
Distribution of Ubiqutin immune-staining in suprarenal gland and renal sections:

Sections of suprarenal glands from natural death cases demonstrated negative ubiquitin cytoplasmic expression in Zona glomerulosa (Fig.5). Variable intensity of ubiquitin immunoexpression were observed according to survival post injury interval; where traumatic deaths with short survival period $(<24 \mathrm{~h})$ revealed moderate ubiquitin immunopositives staining $(++v e)$ in all zones of adrenal cortex (Fig.6). Conversely, severe ubiquitin immunopositives staining $(+++$ ve) in all zones of adrenal cortex were detected in traumatic deaths with long survival period ( $>24 \mathrm{~h}$ ) (Fig.7).

Kidney sections from natural death cases showed focal mild to moderate $(+\underline{v e}$ to $++v e)$ ubiquitin immunopositives staining in distal and collecting tubules while the proximal tubules were negative (Fig.8). Additionally, moderate ubiquitin immunopositives staining (++ve) in $40 \%$ of distal and collecting tubules were observed in traumatic deaths with short survival period $(<24 \mathrm{~h})$ (Fig. 9). However, all traumatic deaths with prolonged survival period $(>24 \mathrm{~h})$ revealed intense positive nuclear and cytoplasmic staining for ubiquitin $(+++v e)$ in more than $90 \%$ of kidney tubules (Fig. 10).

Table (2) demonstrated significant difference of ubiquitin immunoexpression between different types of death in renal distal convoluted tubules and suprarenal cortex; where traumatic deaths showed significant increase in ubiquitin expression compared to natural deaths. However, no significant difference of ubiquitin immunostaining could be detected between blunt and sharp causes of death. Additionally, there was no significant difference of ubiquitin immunostaining in renal proximal convoluted tubules and suprarenal medulla among various causes of death.

Regarding survival period, there was significant positive correlation between survival period and positive ubiquitin immune expression in renal distal convoluted, collecting tubules $(\mathrm{r}=0.461, \mathrm{p}=0.005)$ and suprarenal cortex $(\mathrm{r}=0.459, \mathrm{p}=0.006) \quad$ (Fig. 11).

Table (1): Statistical analysis for comparison between traumatic and natural deaths as regards age, gender\& residence and survival period

\begin{tabular}{|c|c|c|c|c|c|c|}
\hline & \multirow[b]{2}{*}{$\begin{array}{c}\text { Natural death } \\
\mathrm{N}=10 \\
(22.22 \%)\end{array}$} & \multicolumn{2}{|c|}{ Traumatic death } & \multicolumn{2}{|c|}{ ANOVA and Chi-Square tests } \\
\hline & & & 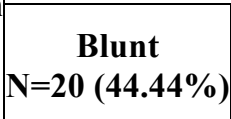 & $\begin{array}{c}\text { Sharp } \\
\mathrm{N}=15 \\
(33.33 \%)\end{array}$ & Test statistic & $P$ value \\
\hline \multirow{4}{*}{$\begin{array}{c}\text { Age } \\
\text { (years) }\end{array}$} & Minimum & 22.00 & 27.00 & 20.00 & \multirow{4}{*}{$\begin{array}{c}F= \\
0.296\end{array}$} & \multirow{4}{*}{0.745} \\
\hline & Maximum & 70.00 & 68.00 & 66.00 & & \\
\hline & Mean & 46.70 & 43.30 & 42.80 & & \\
\hline & SD & 15.99 & 12.04 & 12.81 & & \\
\hline \multirow{4}{*}{ Gender } & \begin{tabular}{l|l} 
& $\mathrm{n}$
\end{tabular} & 8 & 17 & 13 & \multirow{4}{*}{$\begin{array}{c}X^{2}= \\
0.447\end{array}$} & \multirow{4}{*}{1} \\
\hline & Male $\%$ & $80.0 \%$ & $85.0 \%$ & $86.7 \%$ & & \\
\hline & \multirow{2}{*}{ Female $\frac{\mathrm{n}}{\%}$} & 2 & 3 & 2 & & \\
\hline & & $20.0 \%$ & $15.0 \%$ & $13.3 \%$ & & \\
\hline \multirow{4}{*}{ Residence } & \multirow{2}{*}{\begin{tabular}{|l|l|} 
Urban & $\mathrm{n}$ \\
\cline { 1 - 1 } & $\%$
\end{tabular}} & 3 & 5 & 5 & \multirow{4}{*}{$\begin{array}{c}X^{2}= \\
0.579\end{array}$} & \multirow{4}{*}{0.910} \\
\hline & & $30.0 \%$ & $25.0 \%$ & $35.7 \%$ & & \\
\hline & \multirow{2}{*}{\begin{tabular}{l|l|} 
Rural & $\mathrm{n}$ \\
\cline { 2 - 2 } & $\%$
\end{tabular}} & 7 & 15 & 9 & & \\
\hline & & $70.0 \%$ & $75.0 \%$ & $64.3 \%$ & & \\
\hline \multirow{4}{*}{$\begin{array}{c}\text { Survival } \\
\text { period }\end{array}$} & \multirow{2}{*}{\begin{tabular}{|l|l|}
$<1$ day & $\mathrm{n}$ \\
\cline { 2 - 2 } & $\%$ \\
\end{tabular}} & & 14 & 12 & \multirow{4}{*}{$\begin{array}{c}X^{2}= \\
0.078\end{array}$} & \multirow{4}{*}{0.780} \\
\hline & & & $70.0 \%$ & $80.0 \%$ & & \\
\hline & \multirow{2}{*}{\begin{tabular}{|l|l|}
$\geq 1$ day & $n$ \\
\cline { 2 - 2 }
\end{tabular}} & & 6 & 3 & & \\
\hline & & & $30.0 \%$ & $20.0 \%$ & & \\
\hline
\end{tabular}

F: ANOVA test, X2: Chi-Square tests, $p<0.05$ significant

Table (2): Kruskal-Wallis test for comparison between ubiquitin expression in renal and suprarenal gland sections in traumatic and natural deaths

\begin{tabular}{|c|c|c|c|c|c|c|c|c|}
\hline \multirow{2}{*}{\multicolumn{3}{|c|}{ Renal and suprarenal gland tissues }} & \multicolumn{3}{|c|}{ Cause of Death } & \multicolumn{3}{|c|}{ Kruskal-Wallis test } \\
\hline & & & Natural & Blunt & Sharp & $\mathbf{X}^{2}$ & P value & Pairwise comparison \\
\hline \multirow{6}{*}{ Kidney } & \multirow{3}{*}{ Proximal } & Range & $0.00-2.00$ & $.00-2.00$ & $0.00-1.00$ & \multirow{3}{*}{6.76} & \multirow{3}{*}{0.946} & \\
\hline & & Median & 0.00 & 1.00 & 0.00 & & & \\
\hline & & Mean rank & 19.65 & 27.82 & 18.80 & & & \\
\hline & \multirow{3}{*}{ Distal } & Range & \multicolumn{3}{|c|}{$0.00-2.001 .00-3.001 .00-3.00$} & \multirow{3}{*}{14.55} & \multirow{3}{*}{$0.001 *$} & $\mathrm{P} 1=.002 *$ \\
\hline & & Median & 1.00 & 2.00 & 2.00 & & & $\mathrm{P} 2=.001 *$ \\
\hline & & Mean rank & 10.05 & 25.92 & 27.73 & & & \\
\hline \multirow{6}{*}{ Suprarenal } & \multirow{3}{*}{ Cortex } & Range & $.00-1.00$ & $1.00-3.00$ & $1.00-3.00$ & \multirow{3}{*}{23.39} & \multirow{3}{*}{$<0.001 *$} & $\mathrm{P} 1<.001 *$ \\
\hline & & Median & 0.00 & 2.00 & 2.00 & & & $\mathrm{P} 2<.001 *$ \\
\hline & & Mean rank & 6.20 & 28.40 & 27.00 & & & $\mathrm{P} 3=1$ \\
\hline & \multirow{3}{*}{ Medulla } & Range & $0.00-1.00$ & $0.00-2.00$ & $0.00-1.00$ & \multirow{3}{*}{0.901} & \multirow{3}{*}{0.637} & \\
\hline & & Median & 0.00 & 0.00 & 0.00 & & & \\
\hline & & Mean rank & 20.90 & 24.53 & 22.37 & & & \\
\hline
\end{tabular}




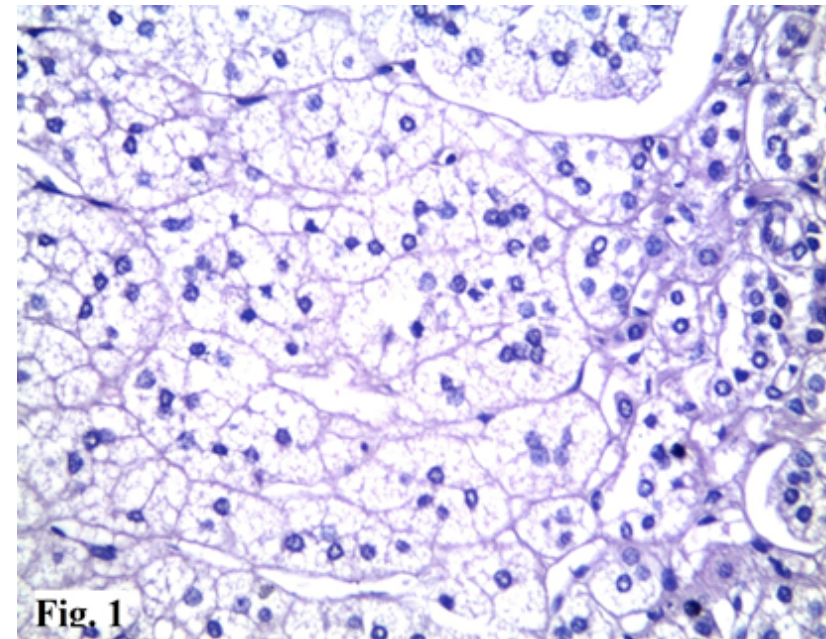

Fig. (1): Photomicrograph of transverse section (T.S.) from suprarenal gland of natural deaths showed apparently normal glandular structure with mild interstitial edema (H\&Ex200).

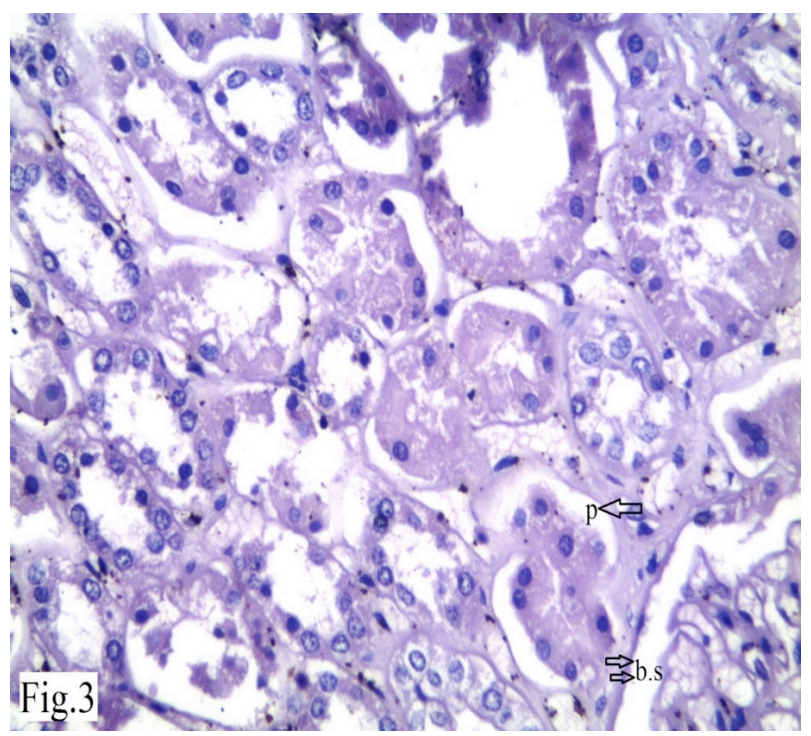

Fig. (3): Photomicrograph of T.S. from kidney of natural deaths showed cloudy swelling of proximal convoluted tubules $(P)$ with widened Bowman capsule (b.s) (H\&Ex200).

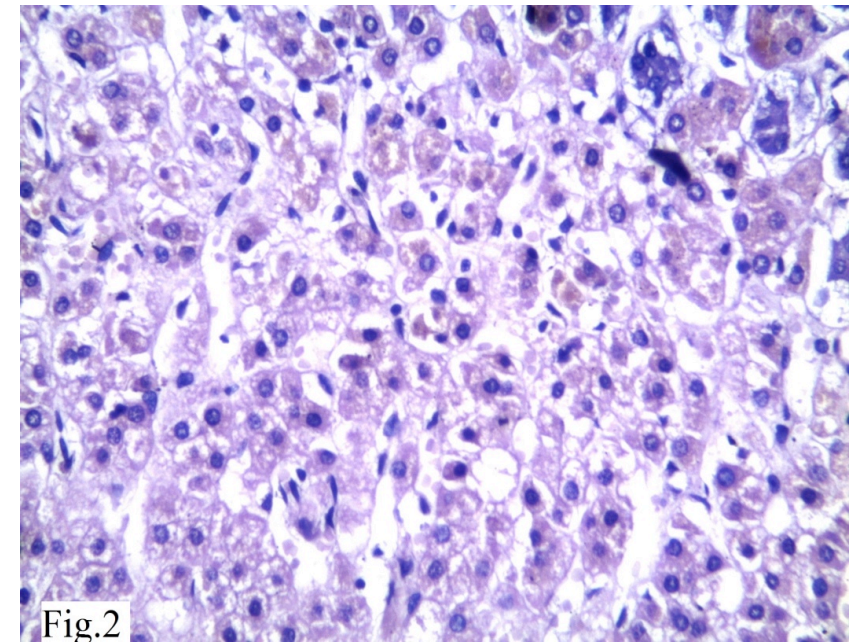

Fig. (2): Photomicrograph of T.S. from suprarenal gland of traumatic deaths showed small size of glandular cells with depletion of its lipid contents (H\&Ex200).

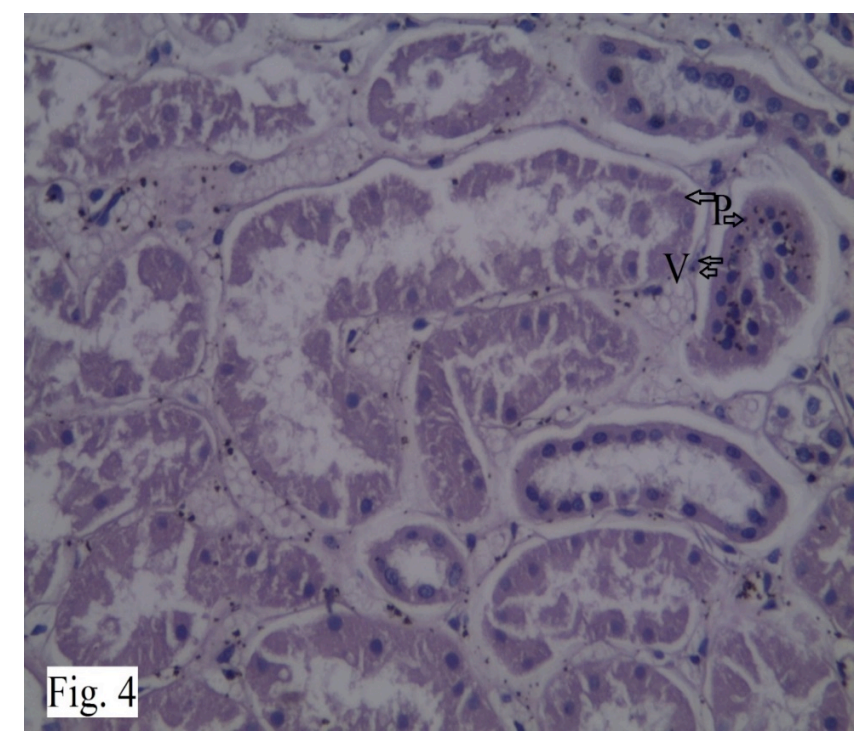

Fig. (4): Photomicrograph of T.S from kidney of traumatic deaths demonstrated an acute tubular necrosis of proximal convoluted tubules (P) and vascular degeneration of distal tubules (V) (H\&Ex200). 


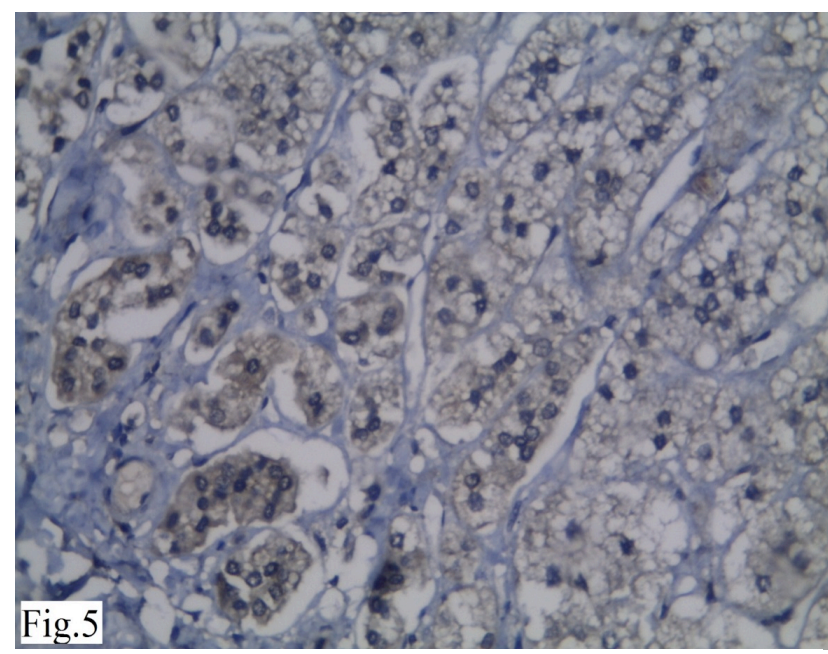

Fig. (5): Photomicrograph of T.S from suprarenal gland of natural deaths showed mild (+ve) ubiquitin cytoplasmic expression in Zona glomerulosa (PAP. X200).

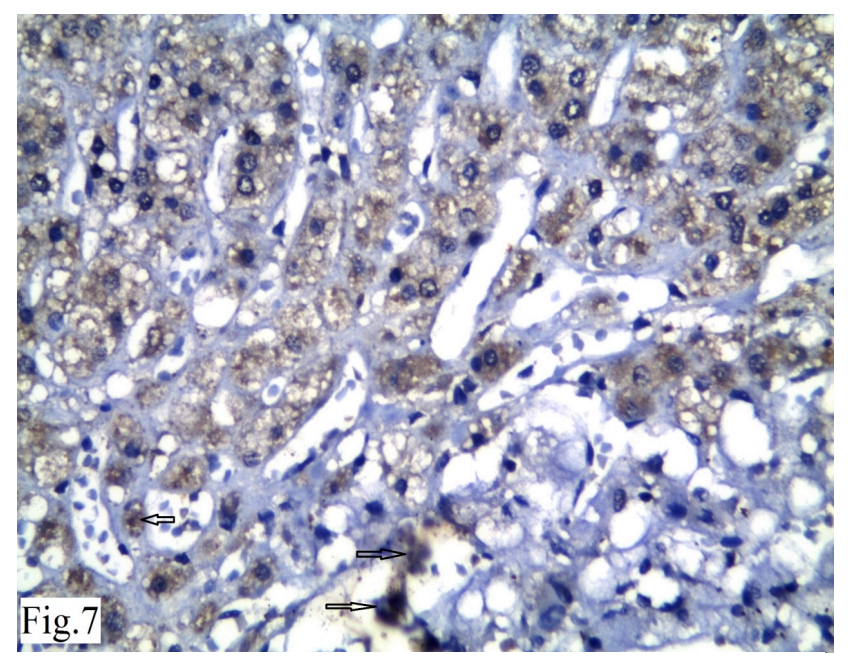

Fig. (7): Photomicrograph of T.S from suprarenal gland of traumatic deaths with $>24 \mathrm{~h}$ survival period showed intensive ubiquitin immunopositivity (+++ve) in all layers of the suprarenal gland (PAPx200)

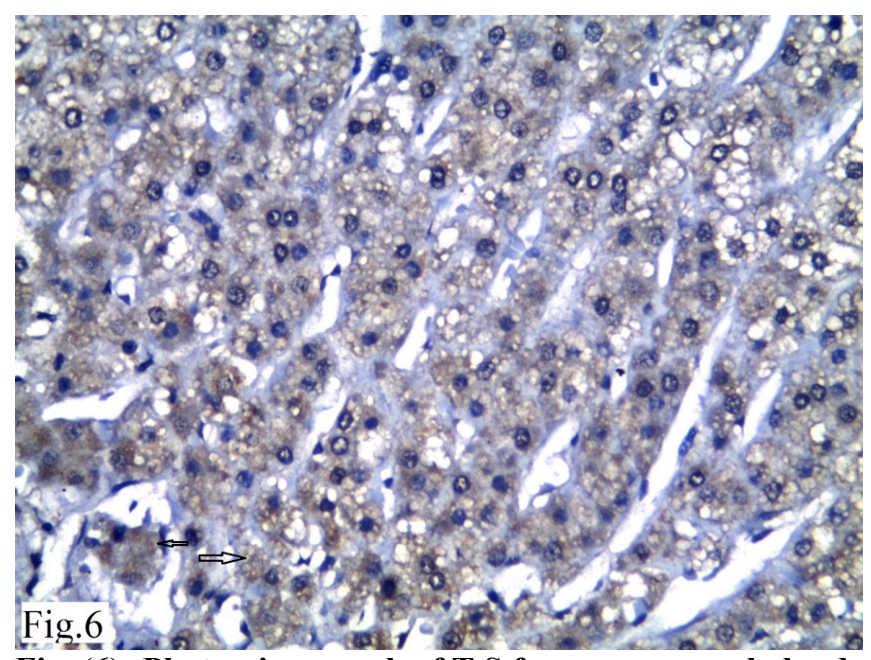

Fig. (6): Photomicrograph of T.S from suprarenal gland of traumatic deaths with $<24 \mathrm{~h}$ survival period showed moderate ubiquitin immunopositivity $(++v e)$ in all adrenal gland zones (PAPx200)\}.

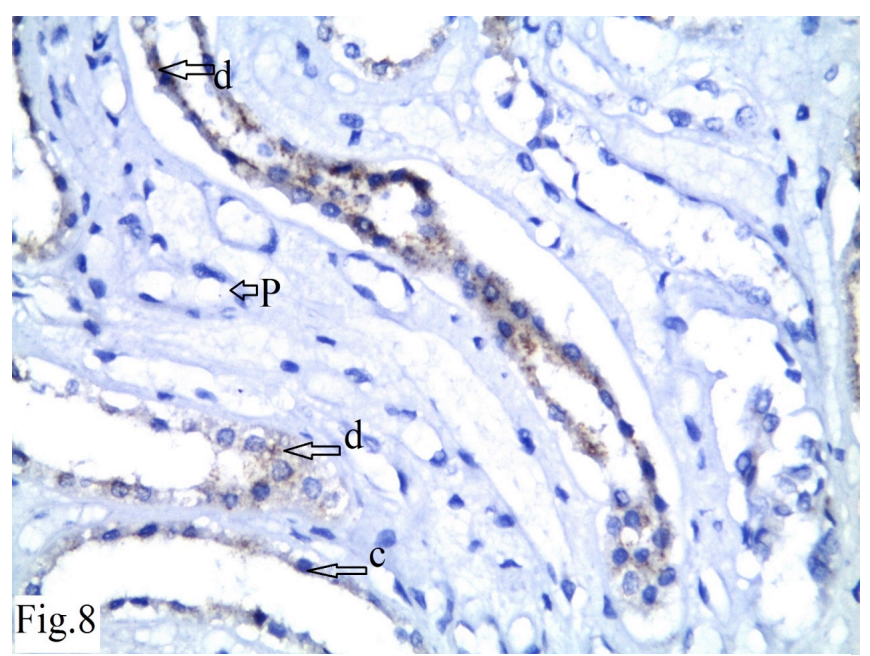

Fig.(8): Photomicrograph of T.S from kidney of natural deaths showed focal mild to moderate Ubiqutin immunostaining $(+$ to $++v e)$ in distal (d) and collecting tubules (C)and negative proximal tubules staining (P) ( PAP. X200). 


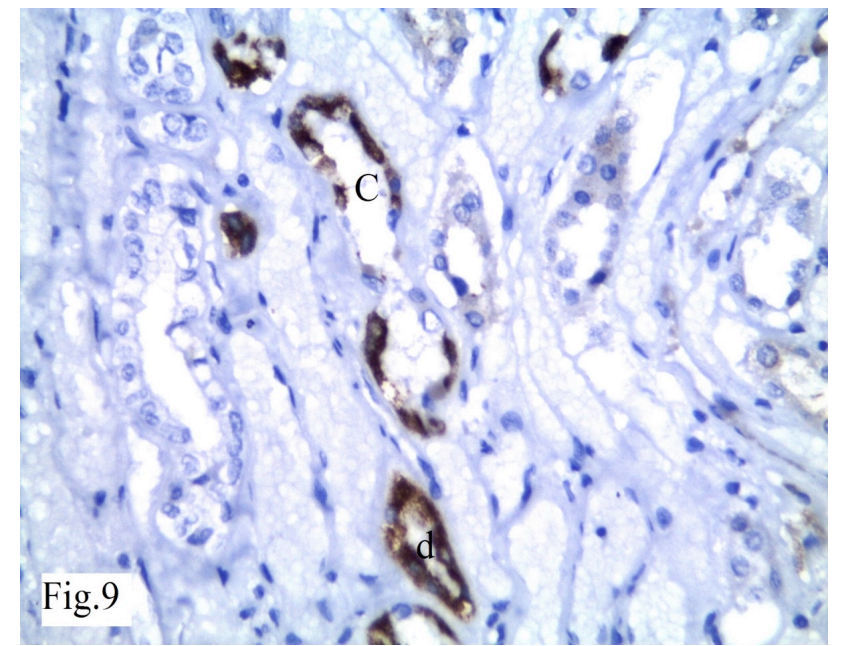

Fig. (9): Photomicrograph of T.S from kidney of traumatic deaths with $<24 \mathrm{~h}$ survival period showed moderate ubiquitin immunopositive staining $(++v e)$ in $40 \%$ of distal (d) and collecting tubules (C) (PAP. X200).

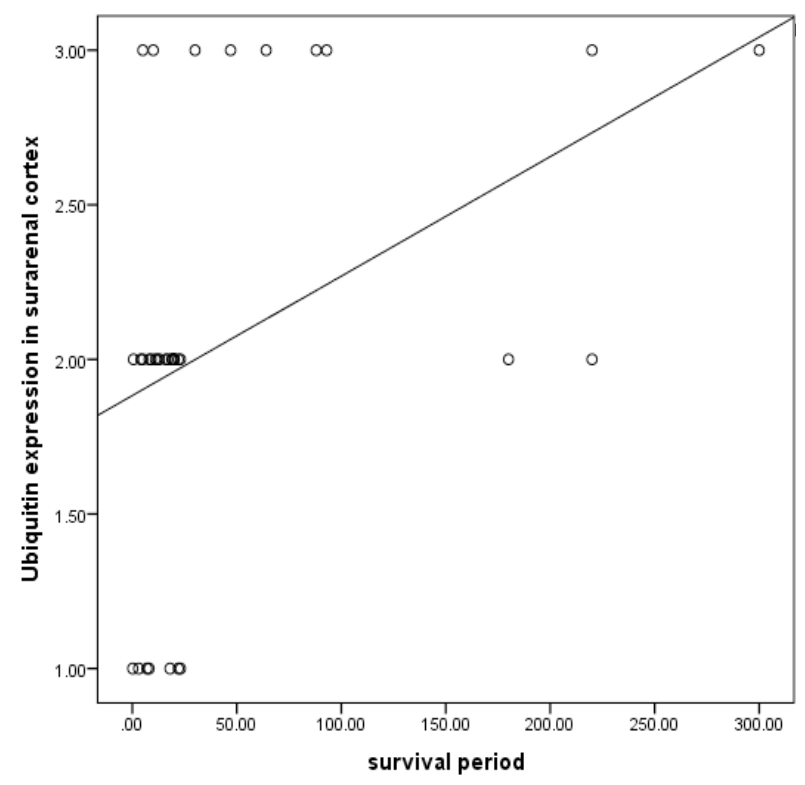

(a)

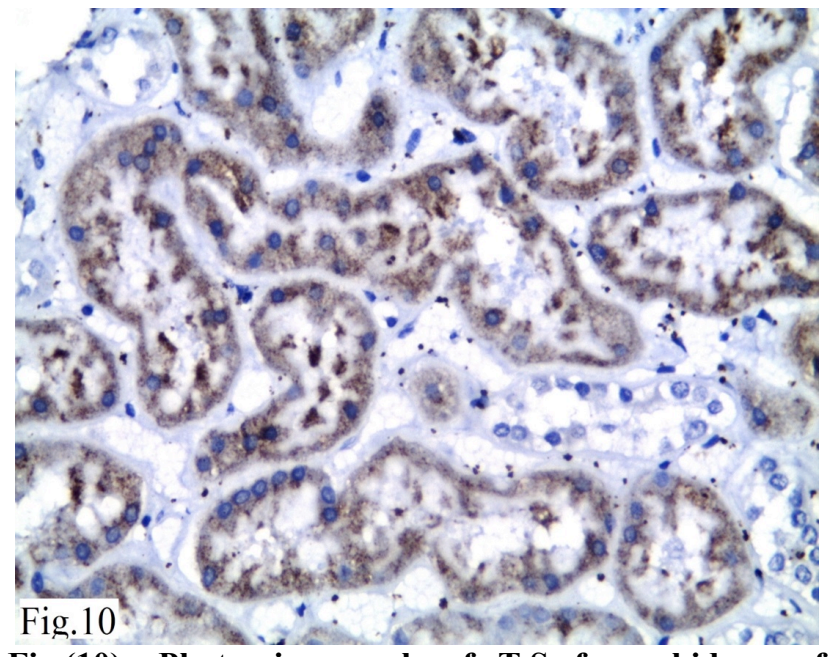

Fig.(10): Photomicrograph of T.S from kidney of traumatic deaths with $>24 \mathrm{~h}$ survival period demonstrated intense positive nuclear and cytoplasmic ubiquitin staining in more than $90 \%$ of distal tubules. *Significant at $p$ value $<0.05 ;$ P1: natural versus blunt; $P 2$ natural versus sharp; P3 blunt versus sharp

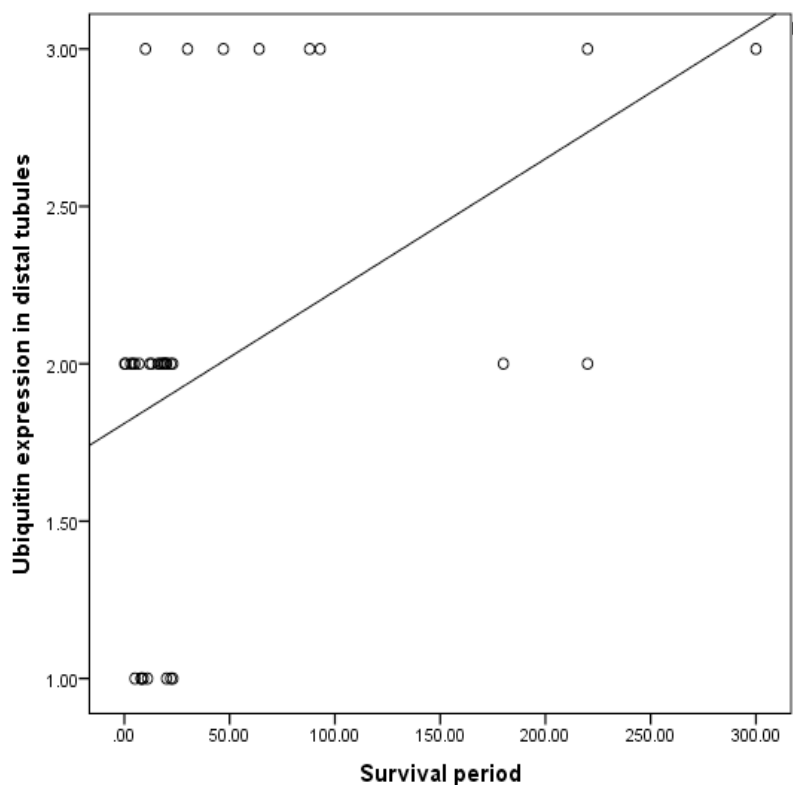

(b)

Fig. (11): Person's correlation between ubiquitin expression and survival period in renal distal tubules (a) and suprarenal cortex (b).

\section{Discussion}

Trauma is still a prominent cause of morbidity and mortality especially in developing countries (Pfeifer et al., 2016). Determination of the cause of death and survival period after trauma is an important task of forensic pathologist in medicolegal cases. It may support the charge and decide whether the injury timing coincides with the prosecution theory or not.However, it is still one of difficult task in current forensic practice (Piette et al., 2011). Immunohistochemical staining may assist the subjective evaluation of medicolegal cases. There are little researches on ubiquitin expression in traumatic deaths. Therefore, the aim of this study was to investigate the expression of ubiquitin in suprarenal and renal tissue in blunt and sharp traumatic deaths.
The current study revealed that, the mean age of subjects was 43.89 years ranged from 20 to 70 years. This finding coincides with study in South USA by Meislin et al. (1997), Italian study by Chiara et al. (2002), American study by Stewart et al. (2003) and Norwegian study by Søreide et al. (2007) who demonstrated that the mean age of traumatic deaths were $42.9,44,42.5$ and 45.8 years respectively.

The majority of cases in the present study were males $(84.4 \%)$ which are in line with Marson and Thomson (2001) who reported that $82.8 \%$ of traumatic cases were males. A systematic review of autopsy studies by Pfeifer et al. (2016) reported that male trauma accounted for $55 \%$ to $88 \%$ from traumatic 
deaths. Moreover, Egyptian injury surveillance in 2009 reported that male predominance in traumatic deaths (71.1\%) (World Health Organization, 2010). Males are obviously at high risk of trauma as they are working outdoors and more exposed to violence and frustrations although females are more susceptible to domestic violence (Kumar et al., 2005; Ali et al., 2007).

The majority of case in the present study were from rural regions $(70.5 \%)$ which is agreement with Boland et al. (2005) and Ali et al. (2007) who demonstrated that the rate of traumatic deaths were significantly higher in rural regions $(66.8 \%$ and $83.55 \%$ respectively). This could be attributed to increase machine related injuries by agriculture work in rural environment (Peek-Asa et al., 2004).

The current study demonstrated that blunt injuries represented $57.14 \%$ of traumatic deaths. This result coincides with Sharma et al. (2005); Ali et al. (2007) and Pang et al.(2008) who reported that 56.9\%, $45.9 \%$ and $53.2 \%$ of traumatic deaths caused by blunt trauma. On country, Min Lo et al. (1992) in New Zeland and Avis (1996) in Canada demonstrated that sharp injuries were more common than blunt injuries. This variation could be attributed to the availability of used weapon in different countries and societies.

The present study revealed that the majority of traumatic cases $(74.3 \%)$ died within 24 hours post injuries. Since the majority of traumatic deaths were occurred within the first 24 to 48 hours of injury (LynSue et al., 2006). Many literatures described a trimodal distribution of deaths (Trunkey and Lim, 1974; Baker et al., 1980). The first peak include $45 \%$ of all deaths that occur within 60 minutes of the injury, $35 \%$ die from one to four hours of the injury (the second peak), while the third peak included late deaths (from many days to weeks after the initial injury) (Lyn-Sue et al., 2006; Pfeifer et al., 2016). The late deaths caused by systemic complications as multiorgan failure, pneumonia or sepsis (Sobrino and Shafi, 2013; Byun et al., 2015).

Examination of renal specimens of traumatic cases by hematoxylin and eosin in the present study revealed acute tubular necrosis in proximal convoluted tubules and vascular degeneration in distal tubules. Acute tubular necrosis (ATN) is a syndrome of intrinsic acute renal failure due to ischemic or toxic insults (Rosen and Stillman, 2008).There are multifactorial causes of post-traumatic renal failure as decreased renal perfusionfrom hypotension, sepsis and rhabdomyolysis secondary to crush injuries (De Abreu et al., 2010).

Additionally, sections from suprarenal gland of all cases of traumatic deaths in the present study showed exhaustion of lipid contents of zona fasciculate with decrease its cell size. The Zona Fasiculata produces glucocorticoids (mainly cortisol), which controls the metabolism of glucose, especially in times of stress (Rabasa and Dickson, 2016). Subsequently, the glucocorticoid secretion in acute stress will increase by continuous stimulation of ACTH, which results in lipid depletion of cells and blood engorgement of cortical sinusoids (Patra et al., 2014).Therefore, lipid depletion of zona fasciculate was demonstrated in antemortem stress caused by trauma, burn and sepsis (Polito et al., 2010 and Marik, 2016).

Ubiquitin is a heat shock protein, which rapidly responds to various types of stress. It has many essential functions as remove or repair denatured cellular proteins that produced by stress and transport them to the proteolytic system (Glickman and Ciechanover, 2002; Flick and Kaiser, 2012). Immunohistochemical staining of ubiquitin expression has been studied in the stress related deaths as fire fatalities, fatal mechanical asphyxia and drowning (Quan et al., 2001a; Quan et al., 2001b).

The present study revealed that ubiquitin immunopositives staining in renal distal convoluted tubules and suprarenal cortex were significantly increased in traumatic deaths compared to natural deaths. This finding coincides with Ishikawa et al. (2007) who demonstrated that ubiquitin immunopositives in renal distal and collecting tubules were significantly higher in deaths from blunt, sharp injuries, fire and hypothermia when compared to natural deaths, poisoning and asphyxia.

Additionally, the significant increase of ubiquitin expression in adrenal cortex in traumatic cases could be attributed to the stress response, which is mediated primarily by the hypothalamic-pituitaryadrenal (HPA) axis with the release of ACTH and cortisol levels (Radley and Sawchenko, 2015; Marik, 2016).The increase in serum cortisol level during stress protects body against developing post-traumatic stress disorder (PTSD) (Cohen et al., 2006). Brouwer et al. (2008) demonstrated ubiquitin-positive intranuclear inclusions in the pituitary and adrenal glands in 100week old mice that could arise from elevated stress hormone levels and disturbed HPA axis physiology. Moreover, Piette et al. (2011) detected ubiquitin immunoreactivity in the human locus coeruleus, which plays a role in the stress response in the hypothalamopituitary-adrenal axis.

The current study did not demonstrate significant difference of ubiquitin expression between blunt and sharp causes of death in renal and suprarenal tissue. It could be attributed to variation of the survival period in the blunt and sharp causes of deaths. In contrary, Quan et al. (2005) and Piette et al. (2011) observed a significant increase in neuronal ubiquitin immunohistochemical expression in blunt trauma compared to sharp trauma $(\mathrm{P}=0.005$ and 0.043 respectively).This finding could be explained by intensive pain that may accompanied with blunt injuries (Quan et al., 2005).

The current study revealed a significant positive correlation between survival period and positive immunoexpression in each of renal distal convoluted tubules and suprarenal cortex. This is in accordance with Ishikawa et al. (2007) who demonstrated survival timedependent of renal tubular ubiquitin immunopositivities in traumatic deaths. Additionally, Piette et al. (2011) determined a significant increase of neuronal ubiquitin immunoexpression with increased agonal stress period. It could be attributed to gradual increase in ubiquitin expression as a cellular response to traumatic painful 
stress (Willis and Westlund, 1997; Quan et al., 2005). Otherwise, polyubiquitination may be a non-specific finding of cellular degeneration as a result of metabolic disorder or organs dysfunction depending on the survival time (Sangerman et al., 2001 and Quan et al., 2005).

\section{Conclusion}

From the current study, ubiquitin immune expression can serve as a stress indicator of traumatic deaths and it may be useful for determination of survival period. Further extended research with large sample size is recommended to support the use of ubiquitin immune expression in determination of survival period in traumatic deaths. Additionally, biochemical indicators of stress (cortisol, catecholamine and adrenocorticotrophic hormones) should be measured to produce a better evaluation of traumatic stress.

\section{References}

Ali E M, Elbakry A A and Ali M A (2007): a study of elderly unnatural deaths in medicolegal autopsies at Dakahlia locality. Mansoura J. Forensic Med. Clin. Toxicol. XV(1):33-43.

Avis SP (1996): Homicide in Newfoundland: A nineyear review. J. Forensic Sci. 41:101-105.

Baker CC, Oppenheimer L, Stephens B et al., (1980): Epidemiology of Trauma Deaths. Am J Surg. 140:144-148.

Boland M, StainesA, Fitzpatrick et al., (2005): Urbanrural variation in mortality and hospital admission rates for unintentional injury in Ireland. Inj. Prev. 11:38-42.

Brouwer JR, Severijnen E, De Jong FH et al., (2008): Altered hypothalamus-pituitary-adrenal gland axis regulation in the expanded CGG-repeat mouse model for fragile $\mathrm{X}$-associated tremor/ataxia syndrome. Psychoneuroendocrinology. 33(6): 863-873.

Byun CS, Park IH, Oh JH et al., (2015): Epidemiology of trauma patients and analysis of 268 mortality cases: trends of a single center in Korea. Yonsei Med J. 56(1):220-226.

Chaudhari SH, Mugadlimath A, Sane Mandar et al., (2013): study of sudden natural deaths in medicolegal autopsies with special reference to cardiac causes. IJCRR. 5(3): 37-42.

Chiara O, Scott JD, Cimbanassi S et al., (2002): Trauma deaths in an Italian urban area: an audit of prehospital and in-hospital trauma care. Injury. 33:553-562.

Cohen H, Zohar J, Gidron Y et al., (2006): Blunted HPA axis response to stress influences susceptibility to posttraumatic stress response in rats. Biol Psychiatry 59:1208-1218.

Dawson-Saunders B and Trapp R (2001): Basic and clinical biostatics. Dawson-Saunders B and Trapp R (eds), 3rd ed., McGrow Hill McGrow Hill Medical Publishing Division, pp: 161-218.

De Abreu KL, Silva Júnior GB, Barreto AG et al., (2010):Acute kidney injury after trauma: Prevalence, clinical characteristics and RIFLE classification. Indian J Crit Care Med.14 (3):121-128.

Flick K and Kaiser P (2012): Protein degradation and the stress response. Semin Cell Dev Biol. 23(5):515-522.
Glickman MH and Ciechanover A (2002): The ubiquitin-proteasome proteolytic pathway: destruction for the sake of construction. Physiol Rev. 82:373-428.

Guler H, Aktas E O, Karali H et al., (2011): The Importance of Tenascin and Ubiquitin in Estimation of Wound Age. Am J Forensic Med Pathol. 32 (1): 83-89.

Hausmann R, Kaiser A, Lang C et al., (1999): A quantitative immunohistochemical study on the time-dependent course of acute inflammatory cellular response to human brain injury. Int J Legal Med. 112: 227-232.

Ishikawa T, Zhu BL, Li DR et al., (2007): Immunohistochemical investigation of ubiquitin and myoglobin in the kidney in medicolegal autopsy cases. Forensic Sci Int. 171(2-3):136-141.

Kleber C, Giesecke MT, Tsokos M et al., (2013): Trauma-related preventable deaths in Berlin 2010: need to change prehospital management strategies and trauma management education. World J Surg. 37(5):1154-1161.

Krug EG, Sharma GK and Lozano R (2000): The global burden of injuries. Am J Public Health. 90(4):523-526.

Kumar S, Jeyasheelan L, Suresh S et al., (2005): Domestic violence and its mental health correlatesin Indian women. British Journal of Psychiatry. 187: 62-67.

Lin Y-H and Wu T (2013): Isolated adrenal hemorrhage after blunt trauma: Case report and literature review. Urological Science. 24: 27-29.

Lyn-Sue J, MRCS, Siram S et al., (2006): Epidemiology of Trauma Deaths in an Urban Level-i Trauma Center Predominantly among African Americans Implications for Prevention. Journal of the National Medical Association. 98(12):1940-1944.

Mahran DG, Farouk O, Qayed MH et al., (2016): Pattern and Trend of Injuries Among Trauma Unit Attendants in Upper Egypt. Trauma Mon. 20; 21(2): e20 967.

Mahran DG, Farouk OA, Qayed M et al., (2013): Hospitalized injuries and deaths in a trauma unit in upper Egypt.Int J CritIllnInj Sci. 3(4):235-240.

Marik PE (2016): Stress Response: Adrenal Function, In: The Stress Response of Critical Illness: Metabolic and Hormonal Aspects. pp 137-152

Marson AC and Thomson JC (2001): The Influence of Prehospital Trauma Care on Motor Vehicle Crash Mortality. J Trauma. 50:917-921.

Meislin H, Criss EA, Judkins D et al., (1997): Fatal Trauma: The Modal Distribution of Time to Death Is a Function of Patient Demographics and Regional Resources. J Trauma. 43(3):433-440.

Min Lo M, Jane CV and Koelmeyer TD (1992): Homicide in Auckland, New Zealand, a 14 year study. Am. J. Forensic Med. Pathol. 13:44-49.

Morris JA, Mucha P, Ross SE et al., (1991): Acute posttraumaticrenalfailure: perspective. J Trauma. 31:1584-1490.

Pang JM, Civil I, Ng A et al., (2008): Is the Trimodal Pattern of Death after Trauma a dated Conceptin 
the 21 st Century? Trauma deaths in Auckland 2004. Injury. 39:102-106.

Patel MB, Earle S A and Majetschak M (2007): Dynamics of Tissue Ubiquitin Pools and UbiquitinProteasome Pathway Component Activities during the Systemic Response to Traumatic Shock. Physiol Res. 56: 547-557.

Patra S, Rath S, Dutta BK et al., (2014): Histological Study of Adrenal Gland In Case Of Suicidal Deaths. IOSR Journal of Dental and Medical Sciences. 13(8): 30-36.

Peek-Asa C, Zwerling C and Stallones L (2004): Acute Traumatic Injuries in Rural Populations. Am. J. Public Health. 94(10):1689-1693.

Pfeifer R, Tarkin IS, Rocos B et al., (2009): Patterns of mortality and causes of death in polytrauma patients--has anything changed?.Injury. 40(9):907911.

Pfeifer R, Teuben M, Andruszkow H et al., (2016): Mortality Patterns, In Patients with Multiple Trauma: A Systematic Review of Autopsy Studies. PLoS One. 11(2):e0148844 (1-9).

Piette MH, Pieters SE and De Letter EA (2011): Evaluation of the agonal stress: can immunohistochemical detection of ubiquitin in the locus coeruleus be useful?.Int J Legal Med.125(3):333-340.

Polito A, De La Grandmaison GL, Mansart A et al., (2010): Human and experimental septic shock are characterized by depletion of lipid droplets in the adrenals. Intensive Care Med. 36:1852-1858.

Quan L, Ishikawa T, Michiue T et al., (2005): Ubiqutin immunoreactivity in the midbrain as a maker of stress to motor nervous systems in fatal injury. Legal Med. 7: 157-163.

Quan L, Zhu BL, Ishida K et al., (2001a)Intranuclear ubiquitin-immunoreactivity of the pigmented neurons of the substantia nigra in fatal acute mechanical asphyxiation and drowning. Int $\mathrm{J}$ Legal Med. 115:6-11.

Quan L, Zhu BL, Oritani S et al., (2001b): Intranuclear ubiquitin immunoreactivity in the pigmented neurons of the substantia nigra in fire fatalities. Int J Legal Med 114:310-315.

Rabasa C and Dickson SL (2016): Impact of stress on metabolism and energy balance. Current Opinion in Behavioral Sciences. 9:71-77.

Radley JJ and Sawchenko PE (2015): Evidence for involvement of a limbic paraventricular hypothalamic inhibitory network in hypothalamicpituitary-adrenal axis adaptations to repeated stress. J Comp Neurol. 523: 2769-2787.
Rosen S and Stillman IE (2008): Acute Tubular Necrosis Is a Syndrome of Physiologic and Pathologic Dissociation J Am SocNephrol. 19: 871-875.

Ross M and Pawlina W (2011): Histology: A Text and Atlas (6th ed.). Lippincott Williams \& Wilkins. pp: 708-780. ISBN 978-0-7817-7200-6.

Roupakias S, Papoutsakis M and Tsikopoulos G (2012): Adrenal injuries following blunt abdominal trauma in children: report of two cases. Ulus Travma AcilCerrahiDerg. 18(2):171-174.

Sangerman J, Kakhniashvili D, Brown A et al., (2001): Spectrin ubiquitination and oxidative stress: potential roles in blood and neurological disorders. Cell MolBiol Lett. 6:607-636.

Sharma BR, Harish MGD and Singh VP (2005): Missed diagnoses in trauma patients vis-a'-vis significance of autopsy. Injury. 36:976-983.

Sixt SU and Dahlmann B (2008): Extracellular, circulating proteasomes and ubiquitin - incidence and relevance. Biochim Biophys Acta. 1782(12): 817-823.

Sobrino J and Shafi S (2013): Timing and causes of death after injuries. Proc (BaylUniv Med Cent). 26(2):120-123.

Søreide K, Krüger AJ, Vardal AL et al., (2007): Epidemiology and Contemporary Patterns of Trauma Deaths: Changing Place, Similar Pace, Older Face. World J Surg. 31:2092-20103.

Stene JK (1990): Renal failure in trauma patients. Crit Care Clin. 6:111.

Stewart RM, Myers JG, Dent DL et al., (2003): Seven Hundred Fifty-Three Consecutive Death in a Level 1 Trauma Center: The Argument for Injury Prevention. J Trauma. 54:66-71.

Trunkey DD and Lim RC (1974): Analysis of 425 Consecutive Trauma Fatalities. J Am CollEmerg Phys.368-371.

Wang XH, Mitch WE (2014): Mechanisms of muscle wasting in chronic kidney disease.Nat Rev Nephrol. 10(9):504-516.

World Health Organization(2010): World Health Organization Injury surveillance: a tool for decision-making: annual injury surveillance report, Egypt, 2009. Egypt: Regional Office for the Eastern Mediterranean, Ministry of Health; Available from:http://www.emro.who.int/dsaf/dsa1087.pdf.

Willis WD and Westlund KN (1997): Neuroanatomy of the pain system and of the pathways that modulate pain. J Clin Neuro physiol. 14:2-31. 


\section{الأهميه الطبية الشرعية للاور التعبيرى للإبيكوتين فى كل من المبلي أنسجة الغدة الكظرية والكلى فى وفيات

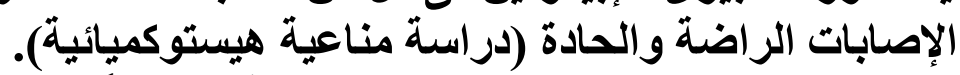

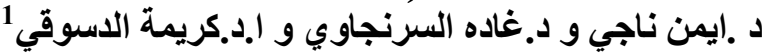

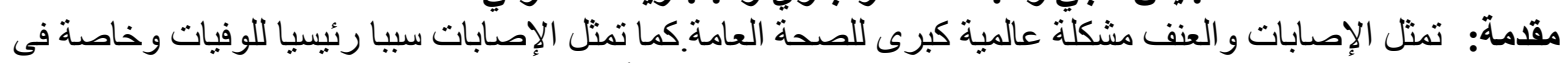

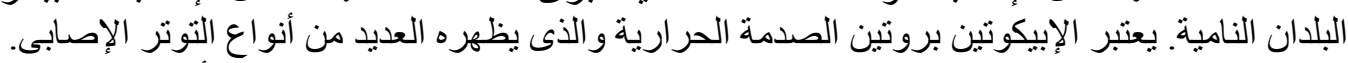

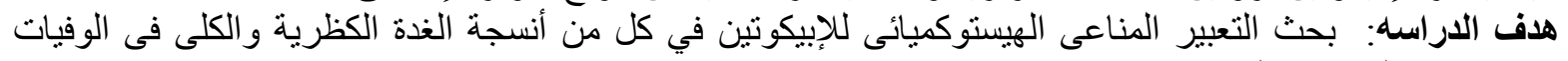
الناشئة عن الإصابات الر اضة و الحادة.

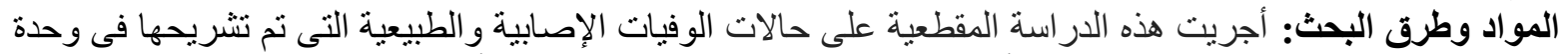

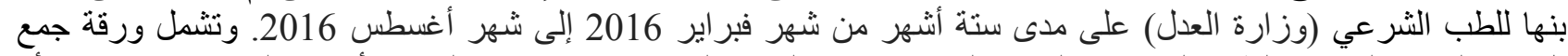

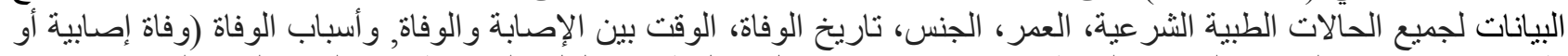

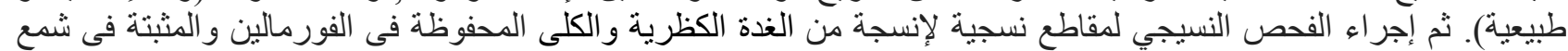

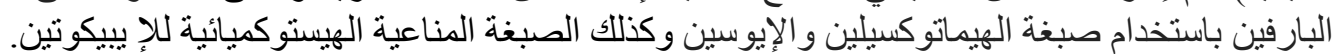

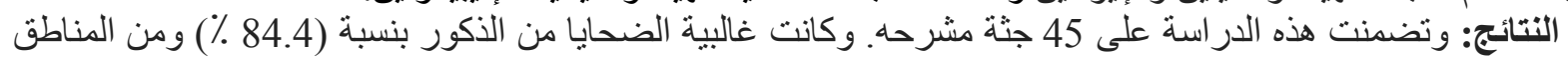

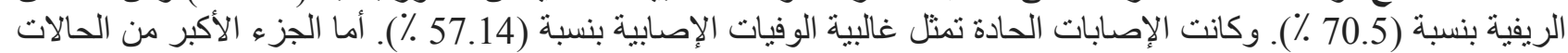

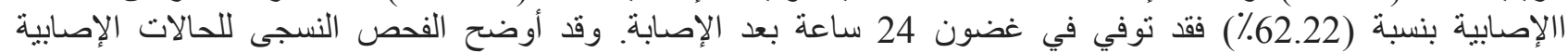

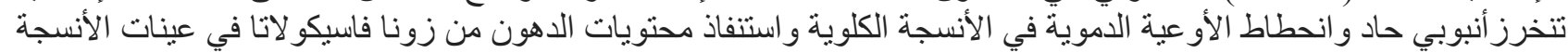

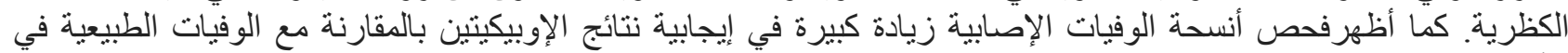

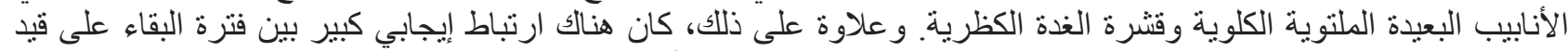

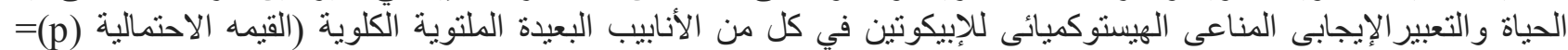

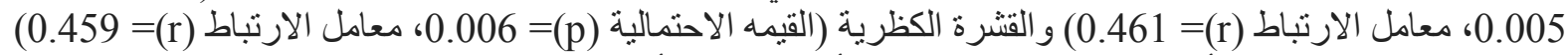

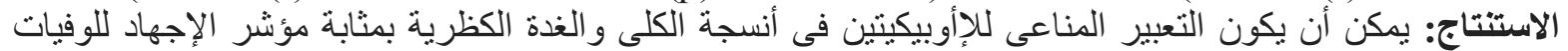

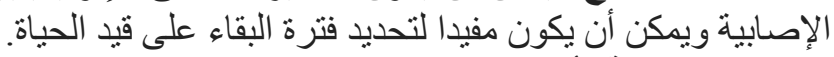
الكلمات الرئيسية: أوبيكيتين، الوفيات الإصابية، التوتئر الإصابِى، الكلى،الغدة الكظرية، الدراسه المناعية الهيستوكميائية، حالات التشريح

1قسم الطب الثر عى و السموم الاكلينيكية و قسم الباثولوجي، كلية الطب، جامعة طنطا 